\title{
Early laparoscopic exploration for acute mesenteric ischemia after cardiac surgery
}

\author{
Sue Hyun Kim', Ho Young Hwang', Min Jung Kim², Kyu Joo Park², Ki-Bong Kim \\ Departments of ${ }^{1}$ Thoracic and Cardiovascular Surgery and ${ }^{2}$ Surgery, Seoul National University Hospital, Seoul National University College of Medicine, Seoul, \\ Korea
}

Acute mesenteric ischemia (AMI) after cardiac surgery is a rare but fatal complication. Early diagnosis and intervention can be lifesaving. We report two cases of patients who underwent early diagnostic laparoscopy for suspicious AMI after cardiac surgery and demonstrated favorable outcomes. An 83-year-old male with severe left ventricular dysfunction underwent off-pump coronary artery bypass grafting. Severe ileus with gaseous distension of the small bowel was developed on the 3rd postoperative day and computed tomographic angiography (CTA) showed pneumatosis intestinalis of small bowel suggestive of AMI. An immediate bedside laparoscopy was performed and it showed preserved perfusion of small bowel. He recovered without complication under supportive medical management. Another 69-year-old male who underwent aortic valve replacement complained of whole abdominal tenderness with severe distension on the 3rd postoperative day. The CTA found segmental non-enhancing bowel wall with air bubbles suggestive of AMI with possible microperforation. A diagnostic laparoscopy demonstrated small-bowel infarction with pus-like fluid collection in the peritoneal cavity. The operation was converted to laparotomy and complete resection of ischemic segments of small bowel was done. He recovered well without any other complications and discharged home on the 35th postoperative day.

Key Words: cardiac surgical procedure; laparoscopy; mesenteric ischemia

Acute mesenteric ischemia (AMI) is a rare but fatal complication after cardiac surgery, with an occurrence rate of $<1 \%$ and mortality rates ranging from $68 \%$ to $100 \%$ [1-4]. Early diagnosis and timely application of appropriate treatment based on a high index of suspicion are important to improve prognosis. However, the definitive diagnosis of postoperative AMI is often difficult because of the variety of clinical presentations and the lack of specificity and sensitivity of diagnostic tools [5]. Herein, we reported two patients who underwent early diagnostic laparoscopy for suspicious AMI after cardiac surgery.

\section{CASE REPORTS}

\section{Case 1}

An 83-year-old man was admitted for severe arteriosclerosis obliterans (ASO) of both legs. Severe left ventricular dysfunction (ejection fraction, 31\%) with two-vessel coronary artery

\section{Case Report}

Received: December 18, 2018

Revised: March 16, 2019

Accepted: April 12, 2019

Corresponding author

Ho Young Hwang

Department of Thoracic and

Cardiovascular Surgery, Seoul

National University Hospital, 101

Daehak-ro, Jongno-gu, Seoul 03080,

Korea

Tel: +82-2-2072-3020

Fax: +82-2-764-3664

E-mail: scalpel@hanmail.net

Copyright $\odot 2020$ The Korean Society of Critical Care Medicine

This is an Open Access article distributed under the terms of Creative Attributions Non-Commercial License (http:// creativecommons.org/li-censes/by-nc/4.0/) which permits unrestricted noncommercial use, distribution, and reproduction in any medium, provided the original work is properly cited. 
disease was diagnosed during preoperative evaluation. $\mathrm{He}$ underwent off-pump coronary artery bypass grafting (CABG) before the surgical correction of ASO. The operation time was 350 minutes, and he was transferred to the cardiac surgical intensive care unit (ICU) in an intubated state. He was extubated on the 1st postoperative day, and intravenous infusions of catecholamine were all discontinued. However, abdominal distension and vague abdominal tenderness without rebound were found on the 3rd postoperative day. Laboratory parameters revealed elevated white blood cell (WBC) count $(26,000 / \mu \mathrm{l})$ and C-reactive protein (CRP) level (20 mg/dl), but fairly normal lactate $(1.1 \mathrm{mmol} / \mathrm{L})$ and procalcitonin $(0.47 \mathrm{ng} / \mathrm{ml})$ levels. Plain abdominal radiography showed ileus with gaseous distension of the small bowel (Figure 1A). On the 4th postoperative day, about $200 \mathrm{ml}$ of blood discharge abruptly appeared in the intraperitoneal drain, which was inserted after harvesting the right gastroepiploic artery during CABG. Computed tomographic angiography (CTA) showed diffuse small-bowel pneumatosis intestinalis and maintained perfusion of the celiac axis and superior mesenteric artery with mesenteric arterial enhancement, which suggested nonocclusive mesenteric ischemia (NOMI) (Figure 1B).

A bedside laparoscopy in the ICU was done 2 hours after the CTA scan. Trocars were placed through a subumbilical (12 $\mathrm{mm}$, camera) and right lower quadrant $(5 \mathrm{~mm})$ incisions. Laparoscopic exploration showed a relatively pinkish distended
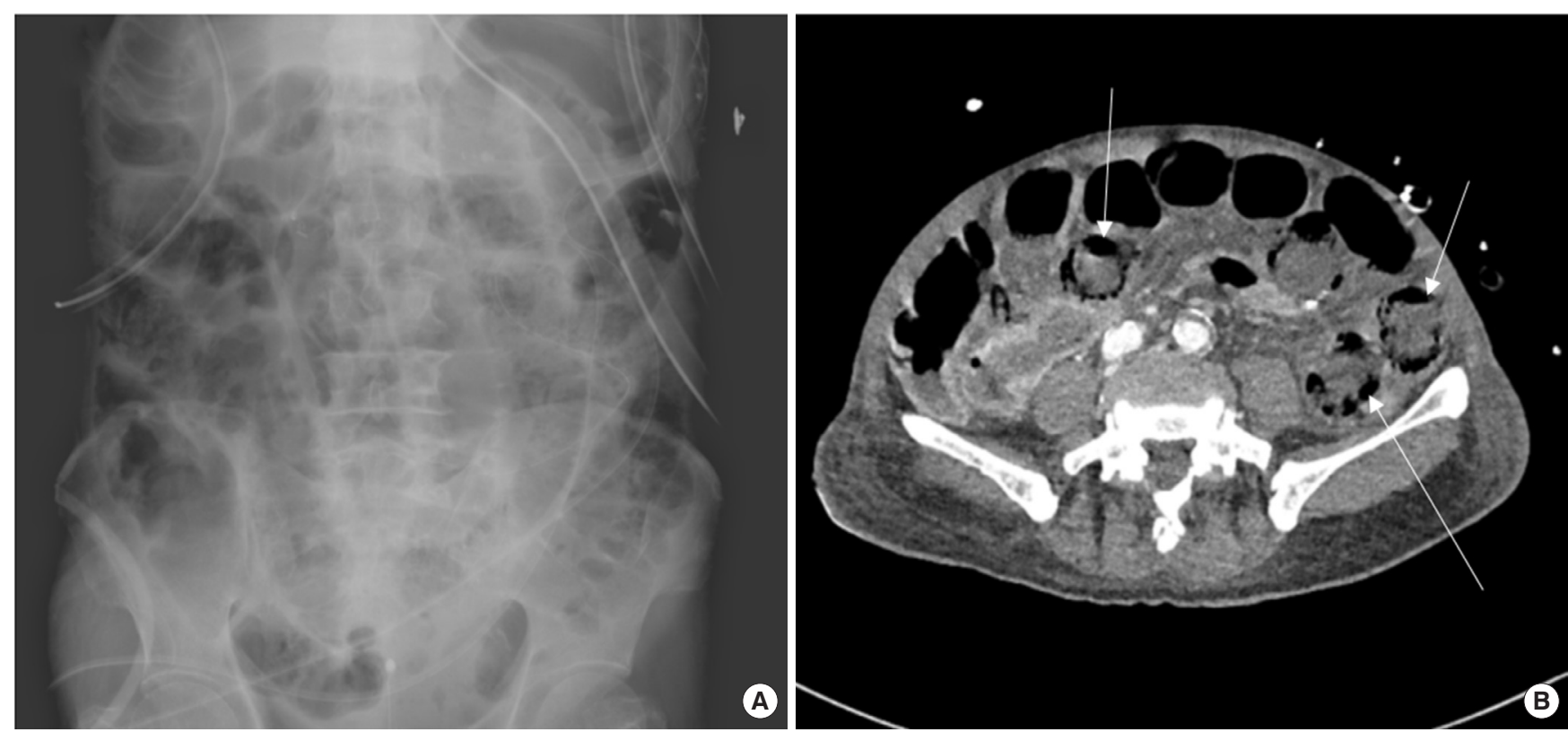

Figure 1. (A) Plain abdominal X-ray showed ileus with gaseous distension of small bowel. (B) Computed tomographic angiography showed multifocal, bubble-like pneumatosis intestinalis of small bowel (arrows).
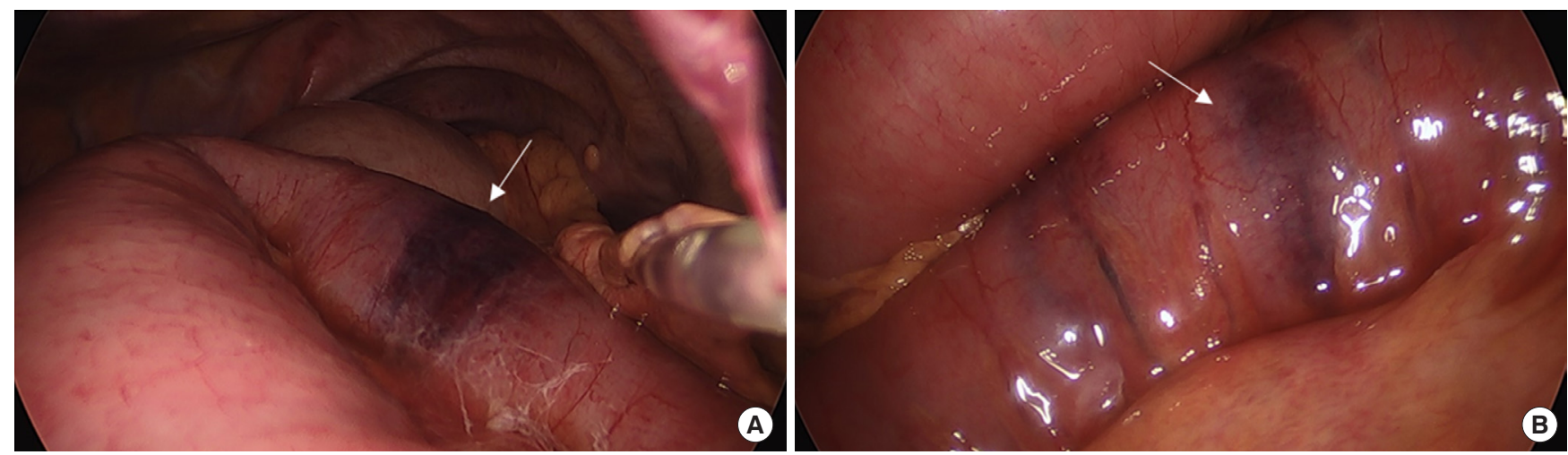

Figure 2. Laparoscopic findings. (A) Relatively pinkish color of distended small bowel without perfusion deficit except a few focal ischemic foci (arrow). (B) Focal ischemic spot (arrow) without evidence of transmural infarction. 
small bowel without definite evidence of perfusion deficit except for a few focal ischemic spots (Figure 2). A Jackson-Pratt drain was placed through the right-lower-quadrant port site for further detection of abnormal fluids.

Postoperative care was focused on the optimization of perfusion through intravenous fluid resuscitation, and any vasoconstrictive agents were avoided. For bowel rest, the patient was placed on nil per os for 14 days followed by careful administration of liquid diet. Third-generation cephalosporin in combination with metronidazole was administered for 14 days to prevent bacterial translocation through the disrupted intestinal mucosa. The patient was transferred to the vascular surgery department for the treatment of ASO without any other complications, at 28 days after CABG.

\section{Case 2}

A 68-year-old man was admitted to our institution for syncope. His medical history included laparoscopic left nephrectomy for a renal pelvis tumor 1 year previously. Severe aortic stenosis was diagnosed, and the patient underwent mechanical aortic valve replacement. The operative time was $200 \mathrm{~min}$ utes. The cardiopulmonary bypass and cardiac ischemic times were 95 and 65 minutes, respectively. He remained intubated and was transferred to the cardiac surgical ICU. He was extubated 5 hours after ICU admission. Oral anticoagulation combined with subcutaneous injection of low molecular weight heparin was started on the 1st postoperative day. He was transferred to the general ward on the next day.

The patient was readmitted to the ICU on the same day be- cause of hemodynamically compromised atrial fibrillation. Abdominal distension and whole abdominal tenderness accompanied by a high fever up to $38.5^{\circ} \mathrm{C}$ were noted on the $3 \mathrm{rd}$ postoperative day. Simple abdominal radiography showed severe gaseous distension of the bowel loops (Figure 3A). CTA showed diffuse dilatation of the bowel loops with multifocal perfusion decrease and pneumatosis intestinalis without evidence of vascular compromise, suggestive of NOMI (Figure $3 B)$. The WBC count was fair $(7,990 / \mu l)$, but the CRP $(31.31$ $\mathrm{mg} / \mathrm{dl})$ and lactate $(6.3 \mathrm{mmol} / \mathrm{L})$ levels were markedly elevated. The general surgery service recommended close observation. On the 5th postoperative day, serum lactate level decreased to the normal range $(1.6 \mathrm{mmol} / \mathrm{L})$, but the WBC count and CRP level were still high $(9,390 / \mu \mathrm{l}$ and $41.97 \mathrm{mg} / \mathrm{dl}$, respectively). The abdominal circumference was decreased by $4 \mathrm{~cm}$; however, the abdomen was still distended, and pain and fever had not subsided. Follow-up CTA was performed, which demonstrated decreased perfusion of the small bowel with segmental nonenhancing bowel wall with air bubbles, suggestive of microperforation (Figure 3C).

The patient was transferred to the operating room and a diagnostic laparoscopy demonstrated small-bowel ischemia and pus-like fluid collection in the peritoneal cavity (Figure 4A). The operation was converted to median laparotomy and complete resection of the ischemic segments of the small bowel, primary end-to-end anastomosis, and creation of a temporary ileostomy were performed (Figure $4 \mathrm{~B}$ ). The length of the remnant small bowel was about $210 \mathrm{~cm}$. Pathologic examination showed transmural infarction with bilateral involvement
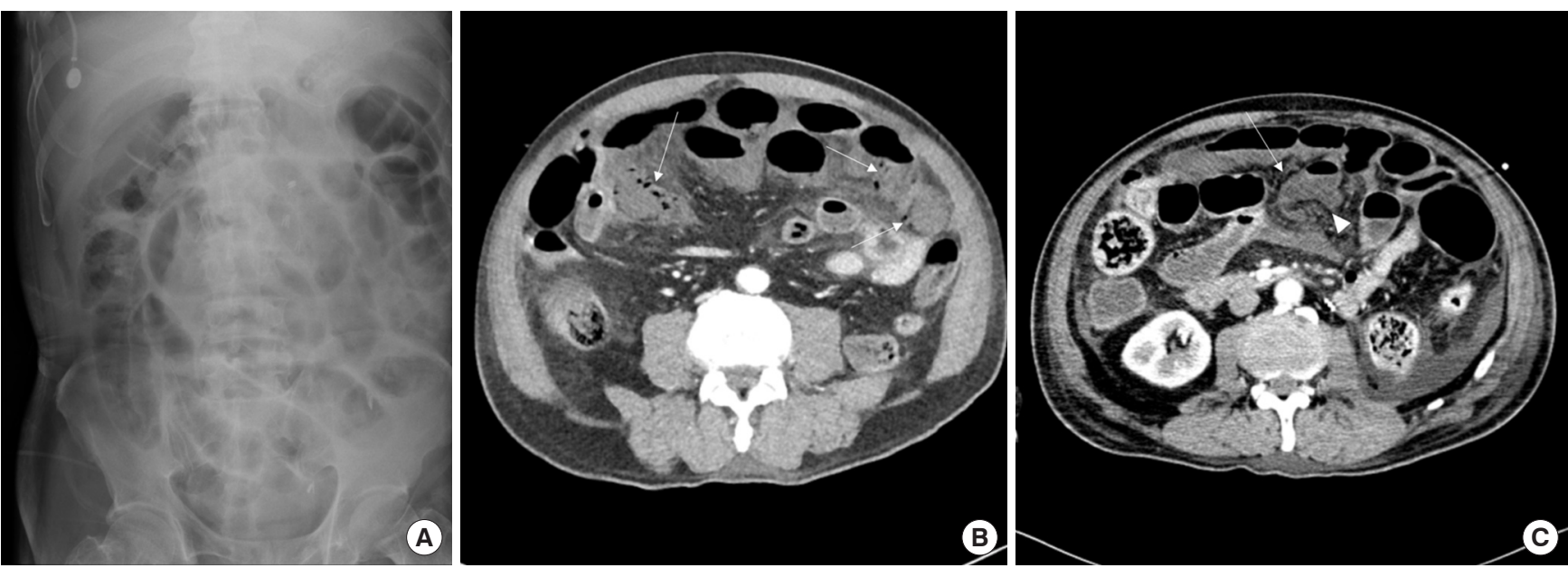

Figure 3. (A) Plain abdominal X-ray showed ileus with gaseous distension of small and large bowel loops. (B) Computed tomographic angiography (CTA) showed multifocal pneumatosis intestinalis of small bowel (arrows). (C) Follow-up CTA showed decreased perfusion of small bowel with segmental nonenhancing bowel wall (arrowhead) suggestive of bowel infarction. Air bubbles with uncertain continuity of bowel segment signify possible combined microperforation (arrow). 

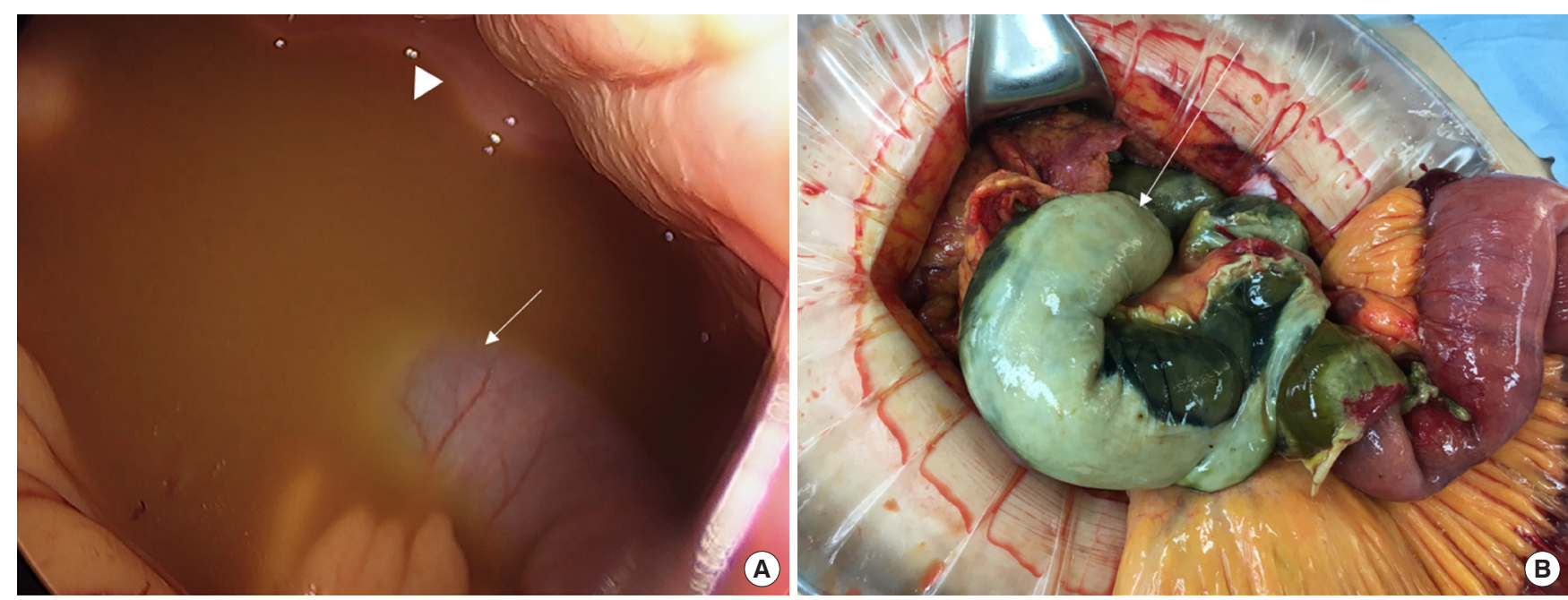

Figure 4. (A) Laparoscopic findings of small bowel ischemia (arrow) and pus-like fluid collection in peritoneal cavity (arrowhead). (B) Ischemic segments of small bowel (arrow) were resected via laparotomy.

of the intestinal resection margins. The patient recovered well after laparotomy and was discharged home without any sequelae on the 35th postoperative day from the initial surgery, with a plan of ileostomy closure at 6 weeks after small-bowel resection.

\section{DISCUSSION}

The AMI is a rare complication after cardiac surgery with a reported incidence of $1 \%$ and $0.2 \%$ to $0.4 \%$ after CABG and heart valve replacement surgery, respectively $[1,2]$. Patients with advanced age and underlying atherosclerosis with risk factors such as perioperative low cardiac output, dehydration, and use of vasopressors are at a high risk of hypoperfusion [5]. The mortality rate of AMI after cardiac surgery has been reported as high as $70 \%$ to $100 \%[1,2]$. The etiologies of AMI are classified into an obstructive origin (arterial thromboembolism and venous thrombosis) and nonobstructive visceral ischemia [6] . The NOMI has been recognized as the predominant form of AMI after cardiac surgery. A previous study [2] reported 52 autopsy cases of bowel infarction after cardiac surgery with cardiopulmonary bypass, with nearly $100 \%$ having a NOMI etiology.

The diagnosis of AMI after cardiac surgery is challenging and often delayed because of nonspecific symptoms, especially in patients under sedation and analgesia in the postoperative ICU setting. There is no single conclusive and discriminative laboratory test for detecting AMI in the early period; serum lactate level increases with disease progression; however, a normal value does not exclude AMI. Owing to the hepatic clearance of lactate through the portomesenteric circulation, lactic acidosis often develops late in the course with extensive transmural infarction [7]. Plain abdominal radiography is nonspecific and shows signs of gaseous distension when bowel infarction has already progressed [6]. Multidetector CTA has a high specificity and sensitivity for detecting AMI (93.3\% and 95.9\%, respectively) according to a meta-analysis [8]. However, CTA findings in NOMI are poorly characterized, with a variable range of bowel wall thickening and enhancement.

Early diagnosis and timely application of appropriate treatment are the key determinants for improving prognosis. Early surgical exploration can be lifesaving, with a $50 \%$ reduction in mortality compared with delayed intervention or conservative management [9]. However, its invasiveness could be a limitation of early application of surgical exploration through laparotomy. In contrast, diagnostic laparoscopy has been suggested as a minimally invasive surgical alternative allowing ready conversion to laparotomy if needed. A previous study [4] reported comparable findings and diagnostic accuracy between laparoscopic and open exploration. Diagnostic laparoscopy is also feasible as a bedside approach in the ICU with the advantage of avoiding time delay for awaiting operating room availability and preventing adverse events during patients transfer who are in critical medical condition, such as on mechanical ventilation or unstable vital sign. However, the routine use of diagnostic laparoscopy in AMI has not been generally adopted [6].

In the present study, we reported two patients in whom AMI was developed after major cardiac surgery. They underwent different treatment pathways based on the diagnostic 
laparoscopic findings; invasive surgical exploration could be avoided in the first case and timely surgical resection could be performed in the second case. In conclusion, early application of diagnostic laparoscopy in cardiac surgical patients with a clinical suspicion of AMI might be an effective strategy to avoid unnecessary laparotomy and to improve outcomes.

\section{CONFLICT OF INTEREST}

No potential conflict of interest relevant to this article was reported.

\section{ORCID}

Sue Hyun Kim

https://orcid.org/0000-0002-7414-5232

Ho Young Hwang

https://orcid.org/0000-0002-8935-8118

Min Jung Kim

https://orcid.org/0000-0002-3105-9633

Kyu Joo Park

https://orcid.org/0000-0002-9134-7426

Ki-Bong Kim

https://orcid.org/0000-0002-4918-7262

\section{AUTHOR CONTRIBUTIONS}

Conceptualization: HYH, KBK, KJP. Data curation: SHK, MJK. Formal analysis: SHK, HYH. Methodology: SHK, HYH. Project administration: SHK, HYH. Visualization: SHK, MJK. Writingoriginal draft: SHK. Writing-review \& editing: HYH, KJP, KBK.

\section{REFERENCES}

1. Mangi AA, Christison-Lagay ER, Torchiana DF, Warshaw AL, Berger DL. Gastrointestinal complications in patients undergoing heart operation: an analysis of 8709 consecutive cardiac surgical patients. Ann Surg 2005;241:895-901.

2. Venkateswaran RV, Charman SC, Goddard M, Large SR. Lethal mesenteric ischaemia after cardiopulmonary bypass: a common complication? Eur J Cardiothorac Surg 2002;22:534-8.

3. Wyers MC. Acute mesenteric ischemia: diagnostic approach and surgical treatment. Semin Vasc Surg 2010;23:9-20.

4. Hackert T, Kienle P, Weitz J, Werner J, Szabo G, Hagl S, et al. Accuracy of diagnostic laparoscopy for early diagnosis of abdominal complications after cardiac surgery. Surg Endosc 2003;17:1671-4.

5. Lorusso R, Mariscalco G, Vizzardi E, Bonadei I, Renzulli A, Gelsomino S. Acute bowel ischemia after heart operations. Ann Thorac Surg 2014;97:2219-27.

6. Tilsed JV, Casamassima A, Kurihara H, Mariani D, Martinez I, Pereira J, et al. ESTES guidelines: acute mesenteric ischaemia. Eur J Trauma Emerg Surg 2016;42:253-70.

7. Demir IE, Ceyhan GO, Friess H. Beyond lactate: is there a role for serum lactate measurement in diagnosing acute mesenteric ischemia? Dig Surg 2012;29:226-35.

8. Yikilmaz A, Karahan OI, Senol S, Tuna IS, Akyildiz HY. Value of multislice computed tomography in the diagnosis of acute mesenteric ischemia. Eur J Radiol 2011;80:297-302.

9. Filsoufi F, Rahmanian PB, Castillo JG, Scurlock C, Legnani PE, Adams DH. Predictors and outcome of gastrointestinal complications in patients undergoing cardiac surgery. Ann Surg 2007;246:323-9. 